\title{
LAS CLAVES DEL ÉXITO DE MUJICA EN LAS PRESIDENCIALES URUGUAYAS DE 2009
}

José Raúl Rodríguez

Daniela Vairo

\begin{abstract}
Resumen
El artículo propone insumos para dilucidar las claves del triunfo de José Mujica en las presidenciales uruguayas de 2009. Bajo una visión de largo plazo se da cuenta de la evolución electoral de los partidos uruguayos así como de las transformaciones en el sistema de partidos. Desde una visión de mediano plazo se coloca el énfasis en la gestión de Vázquez, sus logros y limitaciones, así como su correlato en la opinión pública. La tercera dimensión de análisis, de corto plazo, se centra en variables vinculadas a la conformación de la oferta electoral y al desempeño de los candidatos en campaña. Se concluye que tanto los elevados niveles de aprobación de la gestión del gobierno del Frente Amplio, conjuntamente con una adecuada articulación de la oferta de candidatos actuando en la campaña electoral, acompañados por factores de largo plazo favorables, le permitieron al Frente Amplio retener el gobierno.
\end{abstract}

Palabras clave: Elecciones; Partidos Políticos; Comportamiento Electoral; Campaña Electoral; Opinión Pública.

\begin{abstract}
The article proposes to clarify the key inputs of the victory of José Mujica in the Uruguayan presidential elections of 2009. In a long-term view, presents the evolution of electoral parties and the transformations of the Uruguayan party system. From a medium term view, it focuses on the administration of President Vázquez, its achievements and limitations and its correlate in public opinion. The third dimension of analysis, short term, focuses on variables related to the conformation of the electoral offer and the performance of candidates in the campaign.
\end{abstract}

Keywords: Elections; Political Parties; Electoral Behavior; Campaign; Public Opinion.

\section{Introducción}

El presente trabajo ${ }^{1}$ propone algunos insumos para dilucidar las claves del triunfo de José Mujica en las presidenciales uruguayas del 29 de noviembre de 2009. Nos proponemos analizar los resultados de la elección desde tres dimensiones temporales: de largo, mediano y corto plazo.

La primera dimensión de análisis conlleva una visión de largo plazo. Allí se da cuenta de la evolución electoral de los partidos uruguayos así como las

\footnotetext{
${ }^{1}$ Este trabajo fue presentado en el IV Congreso Latinoamericano de Opinión Pública de WAPOR. Belo Horizonte, 2011.
} 


\section{DOSSIÊ OPINIÃO PÚBLICA, ELEIÇÕES E PARTICIPAÇÃO POLÍTICA NAS DEMOCRACIAS CONTEMPORÂNEAS}

transformaciones en el sistema de partidos. Particularmente, se hace referencia al pasaje del bipartidismo tradicional Partido Nacional (PN) Partido Colorado (PC) al multipartidismo bipolar tras el crecimiento del Frente Amplio (FA) post-dictadura. La bipolaridad PC-PN versus FA es una característica novedosa, que se instaura a partir de las elecciones de 1999, las primeras elecciones con balotaje en el país.

Desde una visión de mediano plazo se coloca el énfasis en la gestión de Vázquez, sus logros y limitaciones, así como su correlato en la opinión pública. El primer gobierno del FA (2005-2009) se destaca por la aprobación de gran parte de su agenda de políticas, al tiempo que por una alta aprobación de su gestión en términos comparativos con los presidentes anteriores. Asimismo, los principales líderes del FA (Vázquez, Mujica y Astori) detentan importantes niveles de popularidad en la opinión pública. Aquí se argumenta que estos factores de mediano plazo favorecieron la posterior victoria de Mujica en 2009.

Finalmente, la tercera dimensión de análisis se centra en variables de corto plazo vinculadas a la conformación de la oferta electoral y el desempeño de los candidatos en campaña. Se hace referencia a las fortalezas y debilidades de los candidatos con énfasis en las fortalezas de Mujica y las debilidades de Lacalle que llevaron a la victoria del primero en el balotaje. Se afirma que tanto la alta popularidad de Mujica como su captación de votantes de izquierda y gran parte de los de centro representaron ventajas para el candidato frenteamplista, mientras que el alto nivel de rechazo a la imagen de Lacalle como su pobre captación de votantes de centro implicaron fuertes limitaciones para el candidato blanco.

La hipótesis central de este trabajo es que tanto los elevados niveles de aprobación de la gestión del gobierno del FA, conjuntamente con una adecuada articulación de la oferta de candidatos expresada en la fórmula Mujica-Astori actuando en la campaña electoral, acompañados por factores de largo plazo favorables, le permitieron al FA retener el gobierno. Como contrapartida, las debilidades del candidato blanco sumado a una errática estrategia de campaña de cara al balotaje no le permitieron librar una elección en segunda vuelta con expectativas de un mejor desempeño electoral. Además del esfuerzo argumentativo, para testear esta hipótesis nos valdremos de datos de opinión pública publicados por las empresas encuestadoras así como de microdatos de una encuesta panel realizada por el Instituto de Ciencia Política (FCS, UdelaR) pocos días antes y después de las elecciones de primera vuelta en octubre de $2009^{2}$. Estos datos son utilizados

\footnotetext{
${ }^{2}$ Es una encuesta panel telefónica realizada por el Instituto de Ciencia Política en el marco del Taller de Procesos Electorales de la Facultad de Ciencias Sociales, Universidad de la República. La primera fase del panel fue realizada entre el 10 y el 18 de octubre de 2009, y la segunda ronda entre el 7 y el 15 de noviembre del mismo año. Para tomar como referencia, la primera vuelta de las elecciones presidenciales se realizó el 25 de octubre y la segunda vuelta el 29 de noviembre de 2009. El análisis, tanto descriptivo como a través del modelo, se realiza sobre la base de datos
} 
tanto para una ilustración descriptiva de los argumentos como para la estimación de un modelo probit de análisis de las dimensiones en conjunto.

\section{Antecedentes en el largo plazo: evolución electoral y sistema de partidos}

Para comenzar es importante aclarar que el sistema para la elección del presidente en Uruguay prevé dos o eventualmente tres instancias electorales. En primer lugar, la realización de elecciones internas, simultáneas y abiertas, con voto no obligatorio, para elegir un único candidato a presidente dentro de cada partido político. Cuatro meses más tarde, tiene lugar la primera vuelta de la elección presidencial, simultánea a las elecciones legislativas. Por último, eventualmente una segunda vuelta (balotaje) si ninguno de los candidatos alcanzara la mayoría absoluta de los votos emitidos ${ }^{3}$.

En un contexto de centralidad de los partidos políticos en la escena nacional, el sistema de partidos uruguayo fue tradicionalmente bipartidista. Desde comienzos de la historia electoral uruguaya y hasta las elecciones de 1971 , los partidos Nacional (PN) y Colorado (PC) retuvieron, en conjunto, alrededor de $90 \%$ del electorado uruguayo. Blancos y colorados no vieron seriamente amenazada su hegemonía bipartidista hasta la irrupción del Frente Amplio (FA) en 1971.

De allí en más, el crecimiento del FA fue sostenido hasta conformarse un sistema de multipartidismo moderado, que se consolida en las elecciones de 1994 (las cuales arrojan un resultado de casi paridad entre los tres partidos) y que luego adquiere una lógica bipolar a partir de 1999. Multipartidismo moderado porque la oferta partidaria por fuera de estos tres partidos no ha logrado desplazar el protagonismo que estos tienen en la definición electoral de los ciudadanos. Luego multipartidismo bipolar, porque en los últimos años se han configurado dos bloques que se disputan y obtienen el gobierno alternativamente, con una base electoral que se encuentra en el entorno de la mitad de la población.

Varios autores refieren al bloque 'tradicional' (del centro a la derecha del continuo ideológico) y al bloque 'progresista' (del centro a la izquierda del continuo ideológico), aunque las denominaciones han sido múltiples. Este multipartidismo bipolar genera lógicas centrípetas de competencia. El primer polo, el tradicional, que está compuesto por el PC y el PN, se caracteriza por tener a uno de los dos partidos como central en cada una de las últimas elecciones, mientras que el otro ocupa un lugar secundario en términos de

\footnotetext{
final, que incluye 604 casos ponderados por intención de voto ajustado a los resultados finales.

3 Para una descripción del sistema electoral uruguayo antes y después de la reforma electoral de 1996, ver Botinelli (1991) y Buquet, Chasquetti y Moraes (1998).
} 


\section{DOSSIÊ OPINIÃO PÚBLICA, ELEIÇÕES E PARTICIPAÇÃO POLÍTICA NAS DEMOCRACIAS CONTEMPORÂNEAS}

votos. En las elecciones de 1999 fue el PC, mientras que en 2004 y 2009 el lugar lo ocupó el PN.

El segundo polo está conformado principalmente por el FA, partido desafiante hasta 1999 y partido de gobierno desde 2004. El FA nace como conglomerado de partidos de izquierda anteriormente existentes y blancos y colorados que se escinden de su partido original. Con el correr de las elecciones, se constituye en un partido tan tradicional como el PN y el PC, con dinámicas similares a las de cualquier partido catch all.

Este proceso de cambio del sistema de partidos se dio de forma gradual y en definitiva estuvo pautado por los cambios en las preferencias de los votantes uruguayos (ver Gráfico 1). Ya en 1971 el FA obtuvo el $18 \%$ de los votos, aumentando cada vez su caudal electoral hasta alcanzar el $52 \%$ en 2004, en detrimento del peso electoral del bloque tradicional. En la última elección de octubre de 2009 el FA desciende levemente su votación en relación a la elección anterior, y se constata un aumento de los votos al PC, disminuyendo el PN.

Gráfico 1 - Evolución elecciones presidenciales en Uruguay (1971-2009, tres principales partidos, en \% de votos válidos)*

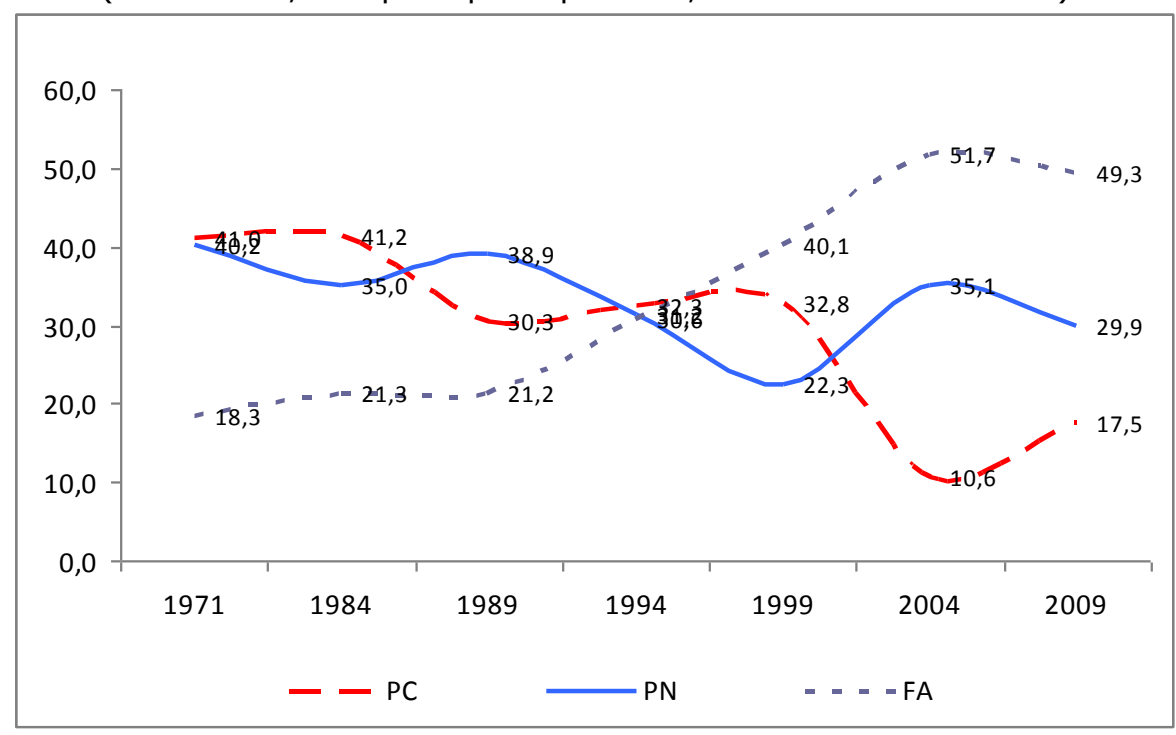

* Primera vuelta

Fuente: Elaboración propia en base a datos del Área Política y de Relaciones Internacionales del Banco de Datos de la FCS

El debilitamiento de los partidos tradicionales ante la opinión pública se relaciona en parte con las reformas estructurales que adoptaron a partir de los años 90, en consonancia con los cambios de inspiración neoliberal que cobraron impulso en Latinoamérica. Aunque siempre dentro de un esquema 
de reformas gradual, el Uruguay impulsó fuertes procesos de desregulación económica que, si bien produjeron resultados favorables en el corto plazo en materia de estabilización macroeconómica, generaron procesos de desindustrialización, precarización laboral, aumento de la desigualdad social y generación de nuevas vulnerabilidades sociales en el mediano plazo ${ }^{4}$.

El cambio en el paradigma de desarrollo probablemente implicó un costo demasiado alto para el bloque tradicional en términos electorales. Si bien los partidos Colorado y Nacional fueron exitosos en materia de aprobación de su agenda gubernativa, la opinión pública les cobró muy caro el hecho de haber impulsado políticas restrictivas en cuanto al papel del Estado. Aunque los uruguayos son críticos de la función pública y del funcionamiento del Estado en general, los distintos estudios muestran que continúan prefiriendo un Estado activo y garante de los derechos económicos y sociales de la ciudadanía, por oposición a un Estado que solamente se limite a regular la economía de mercado. Si bien las reformas llevadas a cabo a partir de los años 90 no revistieron la profundidad observada en otros países de la región, sí fueron lo suficientemente notorias como para habilitar un reposicionamiento de los partidos en el escenario político, dejando la defensa del Estado como promotor del desarrollo en manos del FA, en tanto los partidos tradicionales se asociaron mayormente a la matriz de desarrollo neoliberal.

En este contexto, el FA supo capitalizar de la mano del liderazgo de Tabaré Vázquez las oportunidades electorales que se le presentaron. Si bien Vázquez encarnó la defensa del Estado por oposición al mercado y siempre reivindicó posiciones ubicadas a la izquierda, encabezó un proceso de aggiornamiento ideológico y programático del $\mathrm{FA}^{5}$ en la búsqueda de inhibir el riesgo que podría implicar para buena parte de la opinión pública un primer gobierno de izquierda en Uruguay. Este corrimiento hacia el centro no solamente se expresó en materia programática actuando en campaña electoral sino que también fue parte del sello de su gobierno iniciado en marzo de 2005.

\section{El mediano plazo: la gestión de gobierno de Tabaré Vázquez}

El gobierno de Vázquez se caracterizó por una amplia capacidad de aprobación de su agenda. Algunas medidas centrales que habían sido anunciadas durante su campaña y fueron luego implementadas durante su gobierno son: el restablecimiento de la negociación salarial en los Consejos de Salarios; la implementación del Plan de Emergencia Social (PANES); la

\footnotetext{
${ }^{4}$ Existe coincidencia en señalar que tanto las políticas de apertura arancelaria como las de desregulación laboral generaron un aumento de la desigualdad medida por el Índice de Gini a partir del año 1994. Esta situación comienza levemente a revertirse recién a partir de 2007. Ver PNUD (2008).

${ }^{5}$ Para profundizar en el proceso de moderación ideológica y programática del FA, ver: Garcé y Yaffé (2004).
} 


\section{DOSSIÊ OPINIÃO PÚBLICA, ELEIÇÕES E PARTICIPAÇÃO POLÍTICA NAS DEMOCRACIAS CONTEMPORÂNEAS}

reforma tributaria y aplicación del impuesto a la renta de las personas físicas (IRPF); la creación del Sistema Nacional Integrado de Salud (SNIS); el aumento del presupuesto para la educación al final del período al 4,5\% del PIB; y la política de derechos humanos que implicó una fuerte apuesta a la investigación de los delitos cometidos por los militares durante la dictadura. A estas políticas concretas debe sumarse el mayor crecimiento económico registrado en la historia del país, a tasas acumulativas anuales cercanas al $6 \%$, lo que también tuvo sus consecuencias sobre el abatimiento del desempleo (del $12 \%$ al $8 \%$ en el período), el aumento del salario real y la rebaja de la pobreza del $30 \%$ al $20 \%$ en términos per cápita.

Estas fortalezas del gobierno también tuvieron su correlato político en la opinión pública ${ }^{6}$. Las tres principales figuras del gobierno y del FA fueron las que alcanzaron los mayores niveles de popularidad durante el período (el Presidente Vázquez, el Ministro de Economía Danilo Astori y el Ministro de Ganadería José Mujica). Esta tríada operó como el sostén del gobierno ante la opinión pública, y es también parte de la clave del éxito electoral del FA en 2009 (CANZANI, 2010).

Entre los principales problemas que debió afrontar el gobierno de Vázquez en materia económica se destacan: el sobrecosto energético como consecuencia del alza en los precios del petróleo al comienzo del período; las presiones inflacionarias producidas como consecuencia del crecimiento económico y la baja sostenida en el precio del dólar. En materia de relaciones internacionales: el conflicto con Argentina por la instalación de la planta de celulosa finlandesa sobre el Río Uruguay; la compleja inserción del país en el MERCOSUR; y la discusión que debió librar a la interna del FA en relación al TLC con los EE.UU?' En materia de relacionamiento interno: la inseguridad ciudadana y el hacinamiento carcelario; el dificultoso relacionamiento con la cúpula militar en el marco de la nueva política de derechos humanos. Por último, en materia de gobernabilidad, si bien tuvo que sortear algunos escollos a la interna de su propio partido para aprobar su agenda, contó con un partido fuertemente disciplinado hasta el final del mandato, lo que le permitió aprovechar la mayoría absoluta que ostentaba en ambas cámaras legislativas, además de encarar con éxito el ciclo de interpelaciones parlamentarias llevado adelante por los partidos de la oposición ${ }^{8}$.

En suma, el gobierno de Vázquez muestra un saldo favorable en materia de productividad de políticas y logros económicos y sociales, pero la

\footnotetext{
${ }^{6}$ Para profundizar en el comportamiento electoral de los uruguayos en las últimas tres elecciones ver: VVAA (2000); Buquet (2005); Buquet y Johnson (2010).

7 Este fue un proceso arduo en la interna del partido de gobierno que acabó con la negativa al TLC y con la aprobación de un tratado de protección de inversiones entre Uruguay y EE.UU. (TIFA).

${ }^{8}$ En general hay consenso en que el relacionamiento del gobierno con la oposición no fue bueno, en parte debido al esquema de gobierno de partido mayoritario que inhibió la generación de acuerdos con una base parlamentaria más amplia.
} 
clave estuvo en que fue también un gobierno exitoso ante los ojos de la opinión pública. Más allá de fluctuaciones muy concretas, la evaluación de la gestión del presidente se mantuvo la mayor parte del período por encima del $50 \%$ de aprobación, llegando en el año electoral a valores cercanos al $60 \%$ (ver Gráfico 2) ${ }^{9}$.

Desde que se conocen datos de opinión pública en Uruguay, el caso de Vázquez es notorio por sus elevados y sostenidos niveles de popularidad y aprobación de la gestión. Ninguno de sus predecesores desde la reapertura democrática alcanzó una performance equiparable en este sentido.

Gráfico 2 - Aprobación de la gestión presidencial en los 5 años de gobierno de Vázquez (2005-2009) y comparativo con presidentes anteriores en el año electoral (en \%)

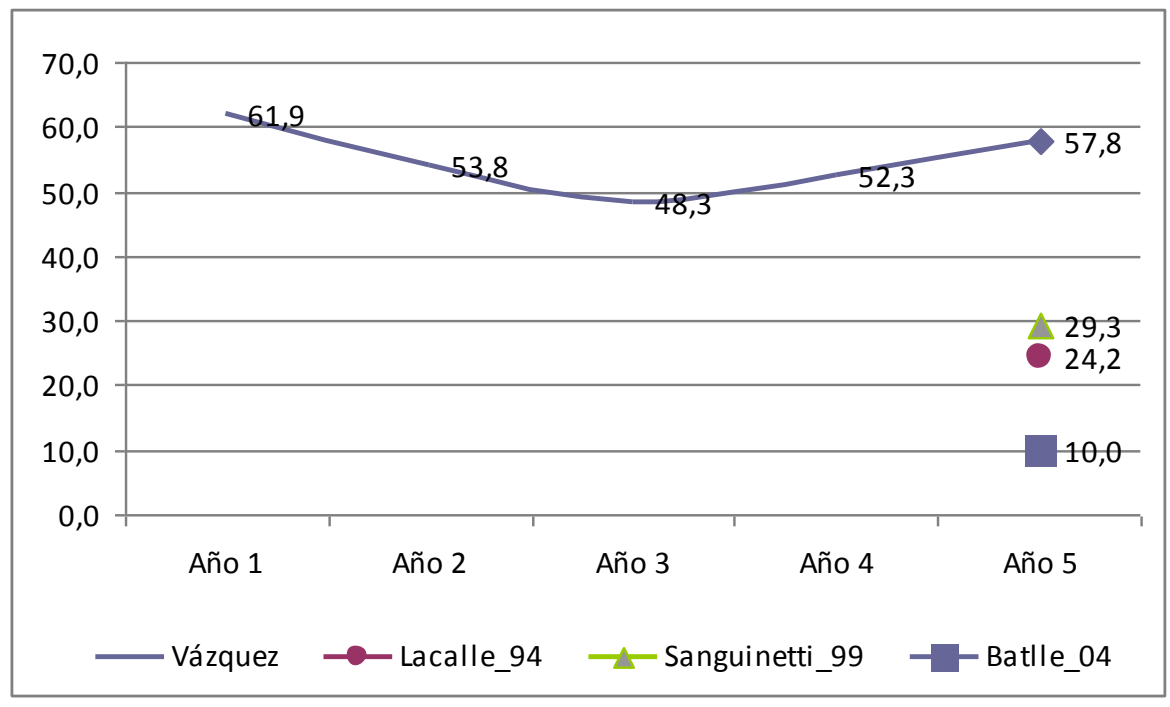

Fuente: Elaboración propia en base a datos del Área Política y de Relaciones Internacionales del Banco de Datos de la FCS

Estos elevados niveles de aprobación se reflejaron en la intención de voto al FA que estuvo en el primer lugar de las preferencias durante todo el período. En particular, en lo que respecta a la segunda vuelta presidencial, se observa una fuerte asociación entre la aprobación de la gestión presidencial y la intención de voto a Mujica. En efecto, a tan solo dos semanas de la elección, $77 \%$ de quienes pensaban que la gestión de Vázquez era buena o muy buena declaraba votar a Mujica, mientras que solamente $15 \%$

\footnotetext{
9 Los valores anuales para el período 2005-2008 corresponden al promedio entre la medias anuales de las mediciones realizadas por las empresas Equipos, Cifra y Factum. Los valores para el año electoral corresponden a promedios de las mediciones anuales realizadas por Equipos.
} 
manifestaba su intención de votar por la fórmula Lacalle-Larrañaga (ver Tabla $1)^{10}$.

Tabla 1 - Intención de voto para el balotaje según aprobación de la gestión presidencial (en \%, octubre de 2009)

\begin{tabular}{l|c|c|c}
\hline & \multicolumn{3}{|c}{ Aprobación gobierno } \\
\hline Intención de Voto & Muy buena/Buena & Ni buena ni Mala & Muy mala/ mala \\
\hline Mujica-Astori & 76.7 & 13.1 & 12.3 \\
\hline Lacalle-Larrañaga & 15.0 & 68.3 & 79.0 \\
\hline En blanco/Anulado & 3.4 & 4.8 & 4.9 \\
\hline Ns/nc & 4.9 & 13.7 & 3.7 \\
\hline Total & $\mathbf{1 0 0 . 0}$ & $\mathbf{1 0 0 . 0}$ & $\mathbf{1 0 0 . 0}$ \\
\hline
\end{tabular}

\begin{tabular}{l|l|l|l|}
\hline Aprobación Gobierno Total & 58.6 & 26.0 & 14.5 \\
\hline
\end{tabular}

Fuente: Elaboración propia en base a los microdatos de Encuesta Panel ICP-FCS 2009.

\section{El corto plazo: la conformación de la oferta electoral y la campaña}

La oferta electoral se compone básicamente de dos aspectos: el candidato y el programa. Estas dos grandes cuestiones sin duda son los andariveles por donde transcurre luego la campaña electoral, además de constituir el marco de referencia sobre el cual los electores definen sus preferencias.

Las elecciones internas del último ciclo electoral se llevaron a cabo en el mes de junio de 2009 y determinaron los triunfos de Mujica (FA), Lacalle (PN), Bordaberry (PC), Mieres (Partido Independiente) y Rodríguez en la novel Asamblea Popular ${ }^{11}$. Estos resultados implicaban ciertos riesgos electorales, fundamentalmente en los casos de los dos principales partidos: el FA y el PN.

En el caso del FA, si bien no podía desconocerse el liderazgo de Mujica y su llegada a nivel de las clases populares, existían dudas sobre su capacidad para captar votantes del centro del espectro ideológico. Esta fue una variable que el propio Vázquez asumió cuando públicamente propuso en 2008 la fórmula Astori-Mujica como la mejor alternativa para retener el gobierno. Los movimientos estratégicos internos del FA que se fueron sucediendo posteriormente arrojaron precisamente el resultado inverso al propuesto por el entonces presidente. La incertidumbre era mayor en un escenario de balotaje, si bien una vez resuelto el dilema de las internas las encuestas tendieron a coincidir en que Mujica era el candidato favorito aún en esa

\footnotetext{
${ }_{10}$ Preguntas en el cuestionario: ¿Cómo calificaría la gestión del actual gobierno? (Muy buena, Buena, Ni buena ni mala, Mala, Muy mala). El próximo 29 de noviembre se llevará a cabo la segunda vuelta de las elecciones presidenciales Ud: ¿Por qué fórmula piensa votar?

${ }^{11}$ Asamblea Popular es un partido escindido del Frente Amplio, ubicado en la extrema izquierda del espectro ideológico. Sin embargo, su escasa votación no le permitió acceder a ninguna banca en el Parlamento.
} 
situación (CANZANI, 2010).

Por su parte, el caso de Lacalle en el PN generaba incertidumbres por su posicionamiento hacia la derecha del espectro ideológico. Se dudaba de su capacidad de captar votantes de centro, lo que lo comprometía notoriamente en un escenario de balotaje. Sin embargo, Lacalle había recuperado el predominio dentro de su partido luego de haberlo perdido en 2004 en manos de su contendor Jorge Larrañaga. Esto lo posicionaba nuevamente en un sitial de privilegio ante la opinión pública que veía cómo había sido capaz de recomponerse en la interna del principal partido de oposición. Su experiencia anterior como presidente entre 1990 y 1995, sumado a cierta incertidumbre generada sobre el desempeño de Mujica como probable presidente, lo colocaban como un candidato con posibilidades.

En ambos casos, los partidos demostraron nitidez para interpretar las reglas de juego y los riesgos en los que incurrían al optar por las candidaturas de Mujica y Lacalle, por lo que rápidamente cerraron sus respectivas fórmulas presidenciales integrando al candidato perdedor. En ambos casos, tanto Astori en el FA como Larrañaga en el PN, le aportaban a la oferta electoral mayores garantías en su búsqueda por captar el voto de centro, operando como contrapeso a lo que podían aparecer como posiciones más extremistas identificadas con los presidenciables.

Sin embargo la primera vuelta del 28 de octubre de 2009 arrojó resultados bien importantes para entender el desenlace definitivo (ver Tabla 2). En primer lugar el FA no alcanzó la mayoría absoluta, lo que aseguraba una segunda vuelta presidencial. No obstante ello, obtuvo la mayoría parlamentaria en ambas cámaras, lo que significó un duro golpe para la oposición en términos de diseñar una estrategia ganadora del bloque tradicional en el balotaje ${ }^{12}$. En tanto, el PN vio bajar su votación de $34 \%$ a $29 \%$ entre 2004 y 2009, lo que le exigía un mayor caudal de voto extrapartidario para poder ganar en el balotaje. Por su parte, el PC se recuperó trepando de un $10 \%$ a un $17 \%$ entre 2004 y 2009 , mientras que el Partido Independiente apenas aumentó su votación llegando a un 2,5\%.

Tabla 2 - Resultado primera vuelta elecciones presidenciales 2009 (en $\%$, votos válidos)

\begin{tabular}{l|c}
\hline Partido & $\%$ \\
\hline Frente Amplio & 49,3 \\
\hline Partido Nacional & 29,9 \\
\hline Partido Colorado & 17,5 \\
\hline Partido Independiente & 2,6 \\
\hline Partido Asamblea Popular & 0,7 \\
\hline Total & $\mathbf{1 0 0 , 0}$ \\
\hline
\end{tabular}

Fuente: Elaboración propia en base a datos de la Corte Electoral.

\footnotetext{
12 Debido al sistema de adjudicación de escaños parlamentarios le fue posible al FA acceder a la mayoría absoluta en ambas cámaras del parlamento.
} 


\section{DOSSIÊ OPINIÃO PÚBLICA, ELEIÇÕES E PARTICIPAÇÃO POLÍTICA NAS DEMOCRACIAS CONTEMPORÂNEAS}

Ante el escenario de un balotaje entre Mujica y Lacalle la principal diferencia consistía en los niveles de popularidad que ambos recogían a nivel de la opinión pública. Más allá de las oportunidades y restricciones que teóricamente podrían atribuírseles a uno y otro, en particular en el escenario del balotaje, la popularidad de Mujica era claramente una ventaja competitiva frente a Lacalle. Mientras Mujica contaba con un $49 \%$ de simpatías y $27 \%$ de antipatías, Lacalle mostraba un saldo negativo de $32 \%$ de simpatías frente a un $47 \%$ de antipatías (ver Tablas 3 y 4 ).

El nivel de popularidad de los candidatos se asocia significativamente a la intención de voto declarada en el tramo final de la campaña hacia el balotaje (ver Tabla 3$)^{13}$. En efecto, la inmensa mayoría de quienes simpatizan con Mujica $(86 \%)$ y de quienes simpatizan con Lacalle $(83 \%)$ manifiestan votar al respectivo candidato en el balotaje. La diferencia está en la amplia ventaja que Mujica le lleva a Lacalle en términos de popularidad a nivel del total de la población.

Tabla 3 - Intención de voto para el balotaje según popularidad de los candidatos (en \%, octubre de 2009)

\begin{tabular}{l|c|c|c|c}
\hline Intención de Voto & $\begin{array}{c}\text { Simpatía por } \\
\text { Mujica }\end{array}$ & $\begin{array}{c}\text { Simpatía por } \\
\text { Lacalle }\end{array}$ & $\begin{array}{c}\text { Antipatía por } \\
\text { Mujica }\end{array}$ & $\begin{array}{c}\text { Antipatía por } \\
\text { Lacalle }\end{array}$ \\
\hline Mujica-Astori & 85.6 & 8.2 & 3.9 & 82.4 \\
\hline Lacalle-Larrañaga & 8.5 & 83.1 & 82.2 & 7.3 \\
\hline En blanco/Anulado & 3.0 & 2.2 & 3.3 & 4.6 \\
\hline Ns/nc & 3.0 & 6.6 & 10.5 & 5.7 \\
\hline Total & 100.0 & 100.0 & 100.0 & 100.0 \\
\hline Total de la población & $\mathbf{4 8 . 8}$ & $\mathbf{3 2 . 9}$ & $\mathbf{2 7 . 3}$ & $\mathbf{4 6 . 8}$ \\
\hline
\end{tabular}

Fuente: Elaboración propia en base a los microdatos de Encuesta Panel ICP-FCS 2009.

Este alto nivel de rechazo a Lacalle constatado en plena campaña electoral hacia el balotaje le imponía una barrera demasiado elevada para ganar la elección. Es probable, como desarrollaremos más adelante, que algunos errores de la campaña lo hayan colocado en esa situación. Pero resulta más atinado suponer que, más allá de puntuales errores de campaña, los valores de popularidad de Lacalle eran deficitarios ya desde el comienzo ${ }^{14}$.

\footnotetext{
13 Pregunta en el cuestionario: "Más allá de su preferencia, ¿cuánta simpatía o antipatía siente usted por los siguientes candidatos? (Mujica, Lacalle). Mucha simpatía, algo de simpatía, ni antipatía ni simpatía, algo de antipatía, mucha antipatía". Se agruparon los datos de mucha simpatía con los de algo de simpatía y los de algo de antipatía con mucha antipatía. La pregunta de intención de voto en el cuestionario es: "El próximo 29 de noviembre se llevará a cabo la segunda vuelta de las elecciones presidenciales Ud: ¿Por qué fórmula piensa votar?".

${ }^{14}$ Si bien la simpatía por Lacalle fue aumentando desde 2005 hasta el año electoral, donde en el primer semestre alcanzó niveles de simpatía cercanos al $40 \%$, la brecha favorable a Mujica siempre estuvo por encima de los 10 puntos porcentuales. Por su parte, Mujica se mantuvo durante la mayoría del período en niveles de simpatía superiores al 50\%. Ver: Canzani (2010, p. 142).
} 
Dicho en otros términos, la figura de Lacalle significaba un problema de oferta para el PN, en el entendido de que no era un candidato con niveles de popularidad competitivos.

Estas diferencias se hacen más notorias al comparar la popularidad de los presidenciables en los distintos estratos de autoidentificación ideológica. En efecto, si bien ambos candidatos son "atractivos" a la interna de sus respectivos segmentos (Mujica en la izquierda y Lacalle en la derecha), resulta significativo observar la fuerte diferenciación que ambos candidatos generan en el segmento de votantes de centro. Mientras que Mujica obtiene un saldo positivo de $+25 \%$, Lacalle en cambio registra un saldo negativo de $22 \%(\text { ver Tabla } 4)^{15}$.

Tabla 4 - Saldos de popularidad de candidatos según segmentos de autoidentificación ideológica (en \%, octubre de 2009)

\begin{tabular}{l|c|c|c|c}
\hline Candidato & Izquierda & Centro & Derecha & Total \\
\hline Mujica & 87 & 25 & -40 & 21 \\
\hline Lacalle & -80 & -22 & 56 & -14 \\
\hline
\end{tabular}

Fuente: Elaboración propia en base a los microdatos de Encuesta Panel ICP-FCS 2009.

Si tenemos en cuenta la distribución del electorado uruguayo en el espectro ideológico (ver Gráfico 3), entonces podemos comenzar a entender la magnitud del diferencial de popularidad entre uno y otro candidato. No solamente el balotaje, sino también la propia dinámica del sistema de partidos uruguayo en el largo plazo, imponen una lucha por el centro del espectro ideológico. Esto ha caracterizado a la mecánica del sistema en tanto el sentido de la competencia ha sido centrípeta desde la reapertura democrática en adelante.

\footnotetext{
${ }^{15}$ El saldo de popularidad es la diferencia entre las opiniones positivas y las negativas (mucha y algo de simpatía menos algo y mucha de antipatía). La pregunta de autoidentificación ideológica en el cuestionario es: "¿En una escala que va desde 0 hasta 10, donde 0 es muy de izquierda y 10 es muy de derecha, en qué valor se ubicaría usted?". La categoría izquierda corresponde a las respuestas de 0 a 3 , el centro es de 4 a 6 y la derecha de 7 a 10.
} 


\section{DOSSIÊ OPINIÃO PÚBLICA, ELEIÇÕES E PARTICIPAÇÃO POLÍTICA NAS DEMOCRACIAS CONTEMPORÂNEAS}

Gráfico 3 - Autoidentificación ideológica de los electores (en \%, octubre de 2009)

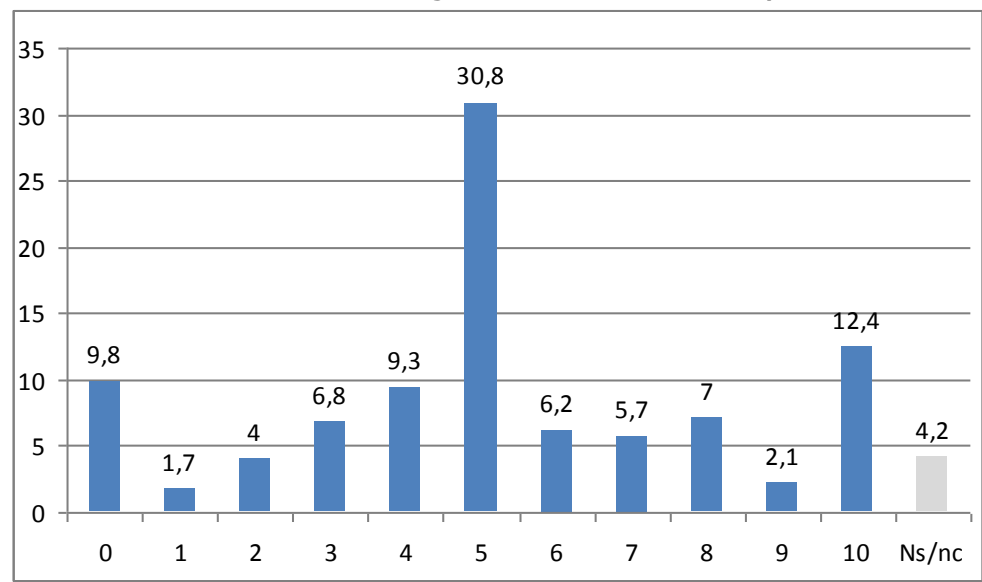

Fuente: Elaboración propia en base a los microdatos de Encuesta Panel ICP-FCS 2009.

Si los partidos, y en particular el FA, han comprendido que deben librar una batalla por los votantes de centro, ¿cómo es posible que no adviertan sobre el nivel de aceptación de la oferta de candidatos en este segmento, que además resulta el más numeroso en término de cantidad de electores? Parte de la explicación puede tener que ver con la lógica del sistema electoral que obliga a los partidos a librar elecciones internas simultáneas y abiertas, con voto no obligatorio.

Esta modalidad estaría generando fuertes restricciones a los candidatos que resultan electos para correrse hacia el centro, fundamentalmente porque el voto no obligatorio produce estímulos para que la participación electoral sea mayor entre los votantes más radicalizados (los más politizados), tanto en la izquierda como en la derecha, desalentando la participación de los votantes de centro. Como señalan Buquet y Piñeiro,

[...] en Uruguay, la posición ideológica de los votantes que participan en las primarias tiene un sesgo hacia ubicaciones más extremas, refrendando los argumentos teóricos de quienes señalan que las primarias suelen beneficiar a candidatos más radicales [...] (BUQUET y PIÑEIRO, 2010, p. $59)$.

Sin embargo, los autores advierten sobre el hecho de que si todos los partidos deciden presentar múltiples candidaturas en la interna, no habría ventajas ni perjuicios para ninguno en particular. Esto es así debido a que en todos los partidos serían seleccionados candidatos únicos más radicales. Distinto es el caso cuando alguno de los partidos define una candidatura única de consenso previo a la interna. Así sucedió con la elección de Vázquez en 2004, donde el FA compareció en la elección interna con candidato único 
evitando una dilucidación de tipo electoral.

Hasta la elección en primera vuelta de octubre de 2009 las campañas de los partidos habían sido bastante previsibles. En el caso del FA, la estrategia consistió en destacar los logros del gobierno a partir de poner a consideración de la opinión pública los resultados de la gestión, y proponer una línea de continuidad y profundización de los "cambios" iniciados por Vázquez. En términos de estrategia de comunicación, la mayor novedad fue la comparecencia en conjunto de Mujica y Astori ante los medios de comunicación, lo que pretendía generar una noción de equipo y despersonalizar la elección, minimizando así las debilidades de Mujica y aprovechando las oportunidades que se vislumbraban por el lado de mostrar a Astori como parte fundamental de la oferta electoral ${ }^{16}$. Por su parte, el PN se dedicó en esta primera etapa a llevar adelante una campaña positiva, en el entendido de que si bien impulsó críticas sobre algunos puntos problemáticos de la gestión (seguridad ciudadana, calidad de la educación, impuesto a la renta, evolución de los precios y del gasto público), intentó posicionarse como una opción para mejorar ("lo podemos hacer mejor").

Sin embargo, la campaña hacia el balotaje simplificó el escenario poniendo a Mujica frente a Lacalle, y en este sentido se observó un comportamiento diferencial de los candidatos y de las estrategias manejadas por uno y otro. Mientras que Mujica continuó apoyándose sobre los mismos argumentos de la primera vuelta, Lacalle incorporó algunas innovaciones que parecen no haberle resultado ${ }^{17}$. En primer lugar se colocó como un candidato suprapartidario. Su campaña giró en torno a una estrategia de comunicación que incorporó códigos que buscaban acercarlo a los otros partidos. Esta estrategia apartidaria, si bien privilegió el acercamiento a los votantes colorados, independientes e indecisos, corría el riesgo de aparecer como poco creíble ante los ojos de la opinión pública ${ }^{18}$.

Al mismo tiempo, cambió el signo de lo que hasta el momento venía siendo una campaña positiva por otra de signo negativo, cuyo epicentro fue el "caso Feldman". Este fue un caso de notoria repercusión mediática donde los secundadores de Lacalle intentaron vincular el hallazgo de un arsenal de armas en una casa ubicada en un barrio de Montevideo con el MLN-

\footnotetext{
${ }^{16}$ Astori fue el primer Ministro de Economía del gobierno de Vázquez y apareció en 2004 y en 2009 como la garantía de estabilidad en el manejo de la macroeconomía y en la moderación de los aspectos programáticos, lo que era un atributo reconocido entre los votantes de centro. Uno de los rasgos salientes de la estrategia del FA fue encargarle desde el inicio del gobierno de Mujica el manejo de la política económica, aun desde su condición de Vicepresidente.

17 Para un mejor detalle de la campaña electoral ver Buquet y Johnson (2010).

${ }^{18}$ La campaña de Lacalle giró en torno a la necesidad de tener un sistema político "equilibrado", que en los hechos era un argumento para contrarrestar la mayoría absoluta que el FA ya había obtenido en el parlamento. De este modo intentó colocar un futuro gobierno dividido como una oportunidad para favorecer el diálogo político. En materia de comunicación, recurrió a colores y frases que intentaban despojarlo de su condición de nacionalista y lo vinculaban con los demás partidos, en particular el Colorado y el Independiente.
} 


\section{DOSSIÊ OPINIÃO PÚBLICA, ELEIÇÕES E PARTICIPAÇÃO POLÍTICA NAS DEMOCRACIAS CONTEMPORÂNEAS}

Tupamaros, otrora fracción del candidato Mujica ${ }^{19}$. No existe constatación empírica de que el "caso Feldman" haya influido de modo significativo en la campaña en un sentido u otro; en definitiva, sí es posible afirmar que no consiguió el efecto buscado por sus ideólogos.

Si bien es cierto que la campaña electoral pudo haber favorecido en mayor medida a la fórmula oficialista, en general los datos de opinión pública disponibles para el período marzo-noviembre de 2009 coinciden en remarcar la fuerte estabilidad de las preferencias de los votantes. Salvo durante el mes de julio donde Lacalle y Mujica prácticamente se encontraban empatados en torno a un $45 \%$ de intención de voto, tanto durante los meses previos como en el tramo posterior al mes de julio, las preferencias por Mujica oscilaron entre el 46 y el $49 \%$ y las de Lacalle lo hicieron entre el 39 y el $41 \%{ }^{20}$.

Finalmente, la fórmula Mujica-Astori obtuvo el $52,4 \%$ de los votos emitidos frente al 43,5\% de Lacalle-Larrañaga (ver Tabla 5). Si comparamos estos resultados con los de octubre - exactamente un mes antes observamos que mientras la fórmula Mujica-Astori ganó 4,4\%, el bloque tradicional (blancos más colorados) perdió 2,4\%. Esto ratifica las dificultades de Lacalle para captar las preferencias del electorado aún dentro del bloque tradicional.

Tabla 5 -Resultados segunda vuelta elecciones presidenciales 2009 (votos emitidos)

\begin{tabular}{l|c|c}
\hline Fórmula presidencial & Votos & $\%$ \\
\hline Mujica-Astori & 1.197 .638 & 52,4 \\
\hline Lacalle-Larrañaga & 994.510 & 43,5 \\
\hline En blanco/anulados & 93.810 & 4,1 \\
\hline Total & $\mathbf{2 . 2 8 5 . 9 5 8}$ & $\mathbf{1 0 0}$ \\
\hline
\end{tabular}

Fuente: Elaboración propia en base a datos de la Corte Electoral

\section{Dimensiones estudiadas y voto a Mujica: un análisis conjunto}

¿Cómo influyen las diversas dimensiones de análisis estudiadas en el voto a Mujica en la segunda vuelta? En este apartado se presentan los resultados de la estimación de un modelo probit $^{21}$ que tiene como variable

19 El "caso Feldman" ocupó buena parte de la agenda mediática en el último tramo de la campaña, donde se intentó traer a un primer plano el pasado guerrillero de José Mujica y todavía más, vincularlo con eventuales movimientos armados en la actualidad. El tema dio lugar a una interpelación al Ministro del Interior por parte de un Diputado de la fracción de Lacalle que terminó sin efectos políticos. Esta estrategia generó discordancias en la propia interna del Partido Nacional.

${ }^{20}$ Existe consenso entre los analistas en que esta paridad fue coyuntural y producto de la buena performance electoral del PN en todo el proceso de la elección interna. Los datos de opinión pública para el período considerado fueron extraídos del Banco de Datos del Área de Política y de Relaciones Internacionales de la Facultad de Ciencias Sociales de la Universidad de la República.

${ }^{21}$ Para la estimación del modelo se utilizará nuevamente la encuesta panel realizada por el ICP en 2009. Los modelos probit son parte de los modelos de respuesta dicotómica. En este caso, el modelo probit explica la probabilidad de votar a Mujica en función de ciertas variables 
dependiente la intención de voto a Mujica para la segunda vuelta de 2009 y como variables independientes los indicadores que hemos venido analizando anteriormente más algunas variables sociodemográficas de control. A continuación (Tabla 6) presentamos la operacionalización de las variables incluidas en el modelo.

Tabla 6 - Operacionalización variables incluidas en el modelo

\begin{tabular}{ll}
\hline Variable & Operacionalización \\
\hline $\begin{array}{l}\text { Intención de voto a } \\
\text { Mujica } \\
\text { (variable dependiente) }\end{array}$ & $\begin{array}{l}\text { Votomujica asume valor 1 cuando el encuestado responde questa. } \\
\text { tiente votar a dicho candidato y valor 0 para cualquier }\end{array}$ \\
\hline $\begin{array}{l}\text { Evaluación de Gestión } \\
\text { del Gobierno }\end{array}$ & $\begin{array}{l}\text { Gestiongobierno asume el valor 1 si el encuestado califica a la } \\
\text { gestión de gobierno de Vázquez como buena o muy buena y } 0 \\
\text { para el resto de las respuestas }\end{array}$ \\
\hline Simpatía por Mujica & $\begin{array}{l}\text { Simpatiamujica asume el valor 1 si el encuestado responde que } \\
\text { le tiene mucha o algo de simpatía y valor 0 para el resto de las } \\
\text { respuestas. }\end{array}$ \\
\hline Simpatía por Lacalle & $\begin{array}{l}\text { Simpatialacalle asume el valor 1 si el encuestado responde que le } \\
\text { tiene mucha o algo de simpatía y valor 0 para el resto de las } \\
\text { respuestas. }\end{array}$ \\
\hline
\end{tabular}

Autoidizquierda asume el valor 1 cuando el encuestado se coloca en los valores entre 0 y 3 de la escala de autoidentificación Autoidentificación ideológica (denominada izquierda) y Autoidcentro asume el valor ideológica 1 cuando el encuestado se ubica en los valores denominados de centro (del 4 al 6 en la escala). Se omite la categoría de derecha (7 al 10 en la escala)

Sexo Mujer asume el valor 1 si el encuestado es mujer y 0 si es
hombre.

Recodificada en tres tramos. Fueron introducidos en el modelo

Edad los tramos de 30 a 64 años (variable De30a64) y 65 años y más (variable De65ymas). Se omite a los jóvenes de 18 a 29 años. Recodificada en tres categorías. Fueron introducidas en el modelo: Secundaria, que asume el valor 1 si el encuestado

Nivel educativo alcanzó el nivel educativo secundario completo o incompleto y Universitaria, que asume el valor 1 si alcanzó el universitario 0 terciario, completo o incompleto. Se omite la categoría de educación primaria.

Lugar de residencia Montevideo asume el valor $1 \mathrm{si}$ el encuestado reside en Montevideo y valor 0 si reside en el interior del país.

Fuente: Elaboración propia

independientes seleccionadas. La categoría omitida de cada variable independiente representa la categoría de referencia para el análisis. Por ejemplo, para la variable "Área Geográfica" se omite la categoría "Interior", por lo que se analiza si existen diferencias significativas entre los montevideanos y los del interior (omitidos) en el voto a Mujica. 


\section{DOSSIÊ OPINIÃO PÚBLICA, ELEIÇÕES E PARTICIPAÇÃO POLÍTICA NAS DEMOCRACIAS CONTEMPORÂNEAS}

En la tabla 7 se presentan los resultados del modelo estimado ${ }^{22}$. Además de los coeficientes, su signo y su significación, se presentan los efectos marginales de las variables que resultaron estadísticamente significativas, como forma de dar cuenta de la magnitud del efecto de las variables independientes sobre la probabilidad de que los encuestados respondan que votarán a Mujica en la segunda vuelta.

Tabla 7 - Coeficientes y efectos marginales, variable dependiente votomujica

\begin{tabular}{|c|c|c|}
\hline Variables & Coeficiente & $\begin{array}{c}\text { Efectos marginales } \\
\mathrm{P}(\text { votomujica } 1)\end{array}$ \\
\hline Gestiongobierno & $1.232^{* \star *}(0,178)$ & $0,447(0.054)$ \\
\hline Simpatiamujica & $1.418^{\star * \star}(0,175)$ & $0,517(0.054)$ \\
\hline Simpatialacalle & $-1.587^{\star * *}(0,207)$ & $-0,530(0.049)$ \\
\hline Autoidizquierda & $0.867^{* * *}(0,268)$ & $0,334(0.095)$ \\
\hline Autoidcentro & $0.432^{\star \star}(0,202)$ & $0,169(0.078)$ \\
\hline Mujer & $0.043(0,174)$ & \\
\hline De30a64 & $-0.378(0,230)$ & \\
\hline De65ymas & $-0.201(0,292)$ & \\
\hline Secundaria & $0.229(0,233)$ & \\
\hline Universitaria & $-0.117(0,254)$ & \\
\hline Montevideo & $0.403^{\star *}(0,173)$ & $0,157(0.066)$ \\
\hline Cons & $-1.493^{\star \star \star}(0,342)$ & \\
\hline
\end{tabular}

Tanto la evaluación de la gestión del gobierno de Vázquez como la simpatía por Mujica y por Lacalle, así como la autoidentificación ideológica del encuestado, están asociadas significativamente con la intención de voto a Mujica. El signo de las relaciones es el esperado en todos los casos. Es decir, la probabilidad de votar a Mujica aumenta cuando el encuestado aprueba la gestión de Vázquez, cuando le simpatiza Mujica, cuando no le simpatiza Lacalle y cuando se autoidentifica como de izquierda o de centro. Estos resultados avalan nuestra hipótesis de que la victoria de Mujica se debe en buena medida a estos factores de mediano y corto plazo. Es fundamental destacar a la luz de estos resultados, la capacidad de captación de votantes de centro por parte de Mujica, dada la distribución ideológica de la ciudadanía que, como veíamos, se concentra en una medida importante en este segmento.

¿Cuál es la fuerza de estas relaciones? Los efectos marginales indican que las variables más fuertemente asociadas son la evaluación de la gestión

\footnotetext{
22 El valor del Pseudo R2 (medida aproximada de bondad de ajuste del modelo) es de 0.6233. La prob>chi2 es 0.000 lo cual nos permite descartar la hipótesis nula de que los parámetros estimados en forma conjunta son 0 .
} 
de gobierno y los dos indicadores de simpatía. La probabilidad de manifestar intención de voto a Mujica aumenta 45 puntos porcentuales entre quienes evalúan positivamente la gestión del gobierno de Vázquez, 52 puntos porcentuales entre quienes tienen simpatía por Mujica, y disminuye 53 puntos porcentuales entre quienes tienen simpatía por Lacalle. Por su parte, quienes se autoidentifican como de izquierda tienen 33 puntos porcentuales más de probabilidad de responder que votarán a Mujica en relación a quienes se autoidentifican como de derecha. El efecto marginal en el caso de los de centro es de 17 puntos porcentuales a favor del candidato frenteamplista.

Con respecto a las variables de control sociodemográficas, tan sólo el lugar de residencia presenta una relación significativa con la variable dependiente. Residir en Montevideo aumenta en 16 puntos porcentuales la probabilidad de votar a Mujica, lo cual va en línea con estudios anteriores que indican que el Frente Amplio tiene mayor votación en Montevideo que en el interior. $\mathrm{Ni}$ el nivel educativo, ni el sexo ni la edad presentan diferencias significativas en relación a la intención de voto a Mujica.

\section{A modo de conclusión}

A la luz del análisis precedente, nos permitimos desarrollar dos argumentos que entendemos claves para entender el triunfo de Mujica en las presidenciales de Uruguay en 2009. En el marco de un sistema de partidos fuertemente institucionalizado, los efectos de mediano y corto plazo no alcanzan para explicar los resultados electorales por sí mismos; pero teniendo en cuenta las reglas para la elección del Presidente y la mecánica bipolar del sistema de partidos en Uruguay, estos asumen singular relevancia en la medida que pueden inclinar la balanza para uno u otro lado.

La primera conclusión es que el diseño de la oferta electoral de los partidos, entendida como la confección de la fórmula, pero principalmente la selección del candidato presidencial, ejerce una influencia decisiva a la hora de definir la competencia electoral. Esta influencia tiene que ver fundamentalmente con el nivel de simpatías que los candidatos recogen en la opinión pública, en particular en el centro del espectro ideológico. Las diferencias observadas a favor de Mujica, pero sobre todo los deficientes resultados de Lacalle en este indicador, nos conducen a concluir que hubo un serio problema de oferta en el PN. Si bien un análisis contrafáctico resulta inconveniente (no podemos probar qué hubiera sucedido en el caso que los blancos hubieran optado por un candidato de mayor popularidad), parece claro que la candidatura de Lacalle implicó una fuerte restricción, la que se vio incrementada por los elevados niveles de popularidad de Mujica.

Independientemente de las consideraciones que partían del supuesto de que Lacalle podía aparecer como un presidenciable más atractivo en comparación a Mujica (quien a priori ofrecía dudas sobre su desempeño como candidato a la presidencia), los datos de opinión pública manejados aquí 


\section{DOSSIÊ OPINIÃO PÚBLICA, ELEIÇÕES E PARTICIPAÇÃO POLÍTICA NAS DEMOCRACIAS CONTEMPORÂNEAS}

indican precisamente lo contrario. Mujica era visto por la opinión pública como un candidato atractivo, mientras que Lacalle era quién ofrecía mayores restricciones. El problema de oferta estuvo del lado del PN; tal vez el FA pudo haber seleccionado un candidato aún mejor, pero en los hechos, los datos de opinión pública sugieren que Mujica fue un candidato suficientemente aceptable para captar las preferencias de la mayoría del electorado.

Una segunda conclusión tiene que ver con la importancia del mediano plazo, entendido como los resultados de gobierno que al final del período se ponen a consideración de la opinión pública. Los uruguayos evaluaron positivamente durante todo el período la gestión del Presidente Vázquez. Aún con algunos altibajos, fundamentalmente a mitad de período, el saldo positivo entre aprobación y desaprobación fue una constante durante los cinco años del gobierno de Vázquez. Más aún, el año electoral encontró al gobierno con crecientes saldos positivos en materia de evaluación de la gestión. ¿Qué razones habría entonces para comprometer la reelección del partido de gobierno? Tal vez las dudas podrían venir de la mano de la dificultad de presentar una oferta electoral atractiva, esto es, un candidato que no fuera atractivo ante la opinión pública. Como explicamos antes, este no fue el caso sino el contrario; el FA presentó un candidato atractivo que supo capitalizar sus ventajas competitivas frente a Lacalle.

Dicho así, parece que la campaña electoral no ha sido determinante en la definición de la elección. Si bien no contamos con información de opinión pública que nos permita incorporar esta dimensión en el modelo propuesto, sí podemos razonablemente suponer que la campaña en todo caso reforzó las tendencias previas. Tanto es así, que el candidato opositor no atacó tanto a los resultados del primer gobierno de izquierda sino que arremetió contra el candidato del oficialismo. En ese intento de centrar la atención en la figura de los candidatos por parte de Lacalle, no pudo evitar errores notorios de campaña; por su parte, la estrategia oficialista logró poner en un primer plano al compañero de fórmula Astori, quién jugó en todo momento como par de Mujica. Tal vez esta estrategia potenció la popularidad de Mujica en el último tramo de la campaña rumbo al balotaje. Sin embargo, queda pendiente estimar con precisión cuáles son los efectos específicos de la campaña electoral sobre el voto. Profundizar el análisis en esta perspectiva puede complementar los hallazgos presentados en este estudio, máxime en un contexto de largo plazo de fuerte estabilidad, donde la competencia interbloques es más bien equilibrada.

En síntesis, una buena gestión, una buena oferta de candidatos, y una buena campaña con pocos errores, parecieran ser las claves de éxito electoral para un gobierno que busca la reelección. En el caso uruguayo, el triunfo de Mujica es un buen ejemplo que cumple con los tres requisitos.

José Raúl Rodríguez es candidato a Magíster en Ciencia Política por la Facultad de Ciencias Sociales, Universidad de la República, es director del Área de 
Opinión Pública y Estudios Sociales de Opción Consultores, Uruguay. Email: jose@opcion.com.uy

Daniela Vairo es candidata a Doctora en Ciencia Política por el Instituto de Estudos Sociais e Políticos (IESP-UERJ), Brasil, se desempeñó como investigadora en Facultad de Ciencias Sociales, Universidad de la República, Uruguay.

E-mail: danielavairo@gmail.com

\section{Referencias}

BOTTINELLI, Oscar. El sistema electoral uruguayo: Descripción y análisis. Working Paper 3, Heildelberg, 1991. 48 p. Disponible en: <http://www2.factum.edu.uy/estpol/sispol/sip90001.html>. Consultado en: 18 dic. 2011.

BUQUET, Daniel (Coord.). Las claves del cambio. Ciclo electoral y nuevo gobierno 2004/2005. Montevideo: Ediciones de la Banda Oriental, 2005. (Colección Política Viva).

BUQUET, Daniel; CHASQUETTI, Daniel; MORAES, Juan A.. Fragmentación política y gobierno en Uruguay ¿un enfermo imaginario? Montevideo: ICPFCS, 1998.

BUQUET, Daniel; JOHNSON, Niki. Del cambio a la continuidad. Ciclo Electoral 2009-2010 en Uruguay. Montevideo: Ed. Fin de Siglo, 2010.

BUQUET, Daniel; PIÑEIRO, Rafael. De las internas a las municipales: los impactos de las reglas electorales en Uruguay. In: BUQUET, Daniel; JOHNSON, Niki (eds.). Del cambio a la continuidad: Ciclo Electoral 2009-2010 en Uruguay. Montevideo: Ed. Fin de Siglo, 2010. p. 45-104.

CANZANI, Agustín. ¿Tipos raros? La lógica de la opinión pública detrás de los resultados electorales 2009. In: BUQUET, Daniel; JOHNSON, Niki (eds.). Del cambio a la continuidad: Ciclo Electoral 2009-2010 en Uruguay. Montevideo: Ed. Fin de Siglo, 2010. p. 135-164.

GARCÉ, Adolfo; YAFFÉ, Jaime. La era progresista. Montevideo: Editorial Fin de Siglo, 2004.

PNUD. 2008, Política, políticas y desarrollo humano en Uruguay. Montevideo: PNUD Uruguay, 2008. 
DOSSIÊ OPINIÃO PÚBLICA, ELEIÇÕES E PARTICIPAÇÃO POLÍTICA NAS DEMOCRACIAS CONTEMPORÂNEAS

VVAA. Elecciones 1999-2000. Instituto de Ciencia Política. Montevideo: Ediciones de la Banda Oriental, 2000.

Texto recebido em 27/06/2011.

Aprovado em 07/12/2011. 